\title{
Evaluation of Monitoring Gases and PM with Low-Cost and Reference Devices at AMS(s) in Belgrade, Serbia
}

\author{
M. Jovašević-Stojanović ${ }^{1}$, D. Topalović ${ }^{1,2}$, M. Davidović ${ }^{1}$, M. Živković ${ }^{1}$ \\ I. Lazović ${ }^{1}$, Z. Ristovski ${ }^{3}$ \\ 1 Vinča Institute of Nuclear Sciences, University of Belgrade, P.O. Box 522, 11001 Belgrade, Serbia \\ 2 School of Electrical Engineering, University of Belgrade, Serbia \\ ${ }^{3}$ Queensland University of Technology, Science and Engineering Faculty, GPO Box 2434, \\ Brisbane QLD 4001, Australia \\ mjovst@vinca.rs
}

\begin{abstract}
Indicative air quality and environmental parameters data with high temporal and spatial resolution including online resource of near real-time and historical data need to be available for citizens. Currently inexpensive air quality sensors have been developed and embedded in small monitoring platforms whose characteristic have been evaluated. This paper shows first results of an evaluation of the performance characteristics of DNET designed low-cost platform under field conditions. They were collocated next to an air monitoring station (AMS) in Belgrade which was a part of the State Air Quality Monitoring Network. Used platforms consisted of electrochemical and infrared sensors for the gaseous pollutants $\mathrm{NO}_{2}, \mathrm{NO}, \mathrm{O}_{3}, \mathrm{CO}, \mathrm{CO}_{2}$, and optical devices for $\mathrm{PM}_{0.5-2.5}$ and $\mathrm{PM}_{2.5-10}$ as well as sensors for monitoring $\mathrm{T}, \mathrm{p}$ and $\mathrm{RH}$. During the three campaigns (Feb-Mar and Oct-Nov 2014), each in duration of 10-15 days, 13 platforms equipped with the same type of Alphasense sensors for gases and DYLOS (USA) monitor for PMs, were tested. The AMS for the first campaign was next to a street with low/medium traffic, while the AMS for the second and last campaign was near the intersection of roads with medium to intense traffic activity. Pilot DNET platforms may be also used for indicative levels of $\mathrm{PM}_{2.5}, \mathrm{PM}_{10}, \mathrm{CO}$ and $\mathrm{CO}_{2}$. The used low-cost devices need to be improved with sensors that will eliminate the influence of $\mathrm{O}_{3}$. The necessity for improving the methodology of calibration of low-cost devices in the field was identified.
\end{abstract}

Key words: air pollution, low-cost sensors, indicative levels, gases, particulate matter, exposure assessment

\section{Introduction}

Atmospheric, urban and indoor air pollution can affect citizen's health in a number of ways. Short-term effects include upper respiratory infections such as pneumonia and bronchitis, while long-term effects include lung and heart diseases and can exacerbate existing conditions such as asthma and emphysema. For such reasons it is important that air quality as well as meteorological data are available for citizens with high temporal and spatial resolution including online resource of near real-time and historical data.

Local, regional and state ambient air quality monitoring networks consist of few monitoring stations, located usual in central zone of the CBD area. These reference and equivalent monitors for ambient PM and gaseous pollutants do not capture spatial gradients in the areas for which they are representative, and cannot provide individualized personal information that is crucial for personal exposure estimation. Beside that there may be a lack of real-time availability of data for citizens. Alternative way may be monitoring indicative levels of the ambient pollutants with the application of miniature and cheap sensors embedded in small devices, so called sensor platforms, for building networks with a much higher spatial resolution and/or use them as personal monitors. So, application of low cost sensor platforms with integrated information and communication tools give an opportunity for:

- adequate spatial resolution and mapping air pollution

- real-time data availability for citizens

- usability data for personal exposure estimation

Prior to the use of sensor pods it is necessary to estimate how accurate or even realistic the 
data collected by these platforms may be, for which period and for which purpose they could be useful.

\section{Material and Method}

During the pilot CITI-SENSE study in Belgrade a DNET static unit, version of EB700++ device, was used. DNET integrated all parts, design electronic, data transmission and data visualization. All measurements are transmitted in real-time over a wireless network and stored on a server.

The DNET static units were equipped with sensors for the following environmental parameters and air pollutants:

- $\quad \mathrm{T}, \mathrm{RH}$ and $\mathrm{p}$ (Alphasense, UK)

- $\mathrm{NO}, \mathrm{NO}_{2}, \mathrm{CO}, \mathrm{CO}_{2}, \mathrm{O}_{3}$ (Alphasense, UK)

- Particulate matter (PM) monitor, two channels for $P M_{0.5-2.5}$ and $\mathrm{PM}_{2.5-10 \mathrm{~mm}}$ (Dylos, USA)

To monitor air pollution, the sensor platforms were equipped with electrochemical-based sensors $\left(\mathrm{CO}, \mathrm{NO}_{2}, \mathrm{NO}, \mathrm{O}_{3}\right)$, infrared $\left(\mathrm{CO}_{2}\right)$ and optical (PMs) sensors (see Fig. 1).
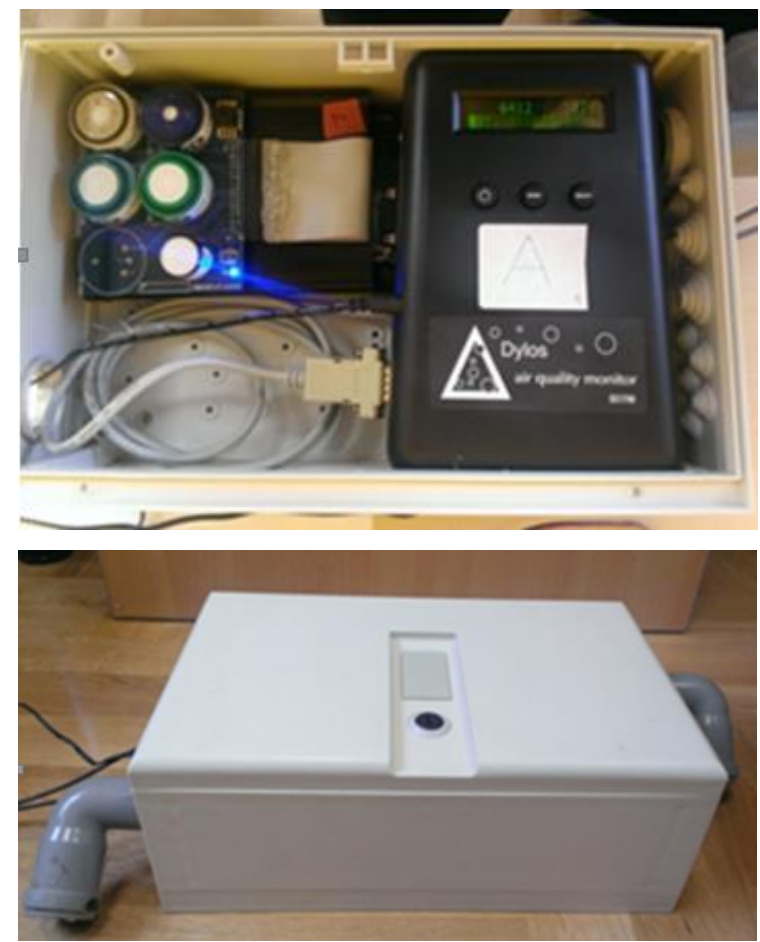

Fig. 1. Photo of DNET EB700++ static platform, inside box and box.

In the framework of the pilot study data collecting campaigns, collecting data with DNET static units collocated to the automatic monitoring stations (AMS) that belong to the State Air Quality Monitoring Network of Serbia running by Serbain Environmetal Protection Agency were performed. AMS's were equipped with reference instruments Thermo analysers for gases, Grimm Model EDM180 for the PM fraction and TESTO monitor for the $\mathrm{CO}_{2}$ data.

Three campaigns were performed with all available static platforms in a duration of 10-15 days. First campaign began in winter, while last finished in autumn as noted in table 1. AMS "Stari Grad" is located near a the city center, at the right side of the river Sava and Danube, and next to the street with low/medium traffic, elementary school, museum and a restaurant. AMS "Novi Beogard" is located at the left side of the river Sava, near to the intersection of roads with medium to intense traffic activity.

Tab. 1: Timeline of DNET pilot platforms collocation at AMS during the CITI-SENSE pilot study

\begin{tabular}{|c|c|c|c|}
\hline $\begin{array}{c}\text { Pilot } \\
\text { campaign }\end{array}$ & Period & Station & Platform \\
\hline \multirow[b]{2}{*}{ First } & 28.02.2014 & \multirow[b]{2}{*}{ Stari Grad } & \multirow[b]{2}{*}{$\begin{array}{c}13 \\
(1-13)\end{array}$} \\
\hline & $\begin{array}{c}17.03 .2014 \\
\text { (15 days) }\end{array}$ & & \\
\hline \multirow[b]{2}{*}{ Second } & 17.03 .2014 & \multirow[b]{2}{*}{$\begin{array}{c}\text { Novi } \\
\text { Beograd }\end{array}$} & 12 \\
\hline & $\begin{array}{c}01.04 .2014 \\
\text { (15 days) }\end{array}$ & & $\begin{array}{l}(1-10 \\
12-13)\end{array}$ \\
\hline Last & $\begin{array}{c}23.10 .2014 . \\
02.11 .2014 \\
(10 \text { days })\end{array}$ & $\begin{array}{c}\text { Novi } \\
\text { Beograd }\end{array}$ & $\begin{array}{c}10 \\
(2-10, \\
13) \\
\end{array}$ \\
\hline
\end{tabular}

\section{Results and Discussion}

Once the monitoring campaign was completed, data from the AMS instruments and Testo monitor were compared to determine how they correlate with the reference instruments.

A filtering of the signals from the platforms by using a smooth function was first performed. The description of the smoothing method together with examples of simplified Matlab codes is described by Gracia (2010). This allows fast, automatic and robust smoothing of signals with arbitrary dimensions, followed by conversion of the count to mass concentration on the basis of approximative, literature data for PMs. The smooth function provides an option to set the input parameters of smoothness with which it is possible to control the filtering process. By increasing the smoothness, the correlation coefficient between the signals from the low-cost sensors and reference monitors has improved.

Figures 2-4 present the Pearson correlation coefficient for gases and PMs between low-cost sensors and reference sensors for each of the monitoring campaigns.

Sensors for environmental parameters, temperature, pressure and humidity show the best results and are the most uniform. 


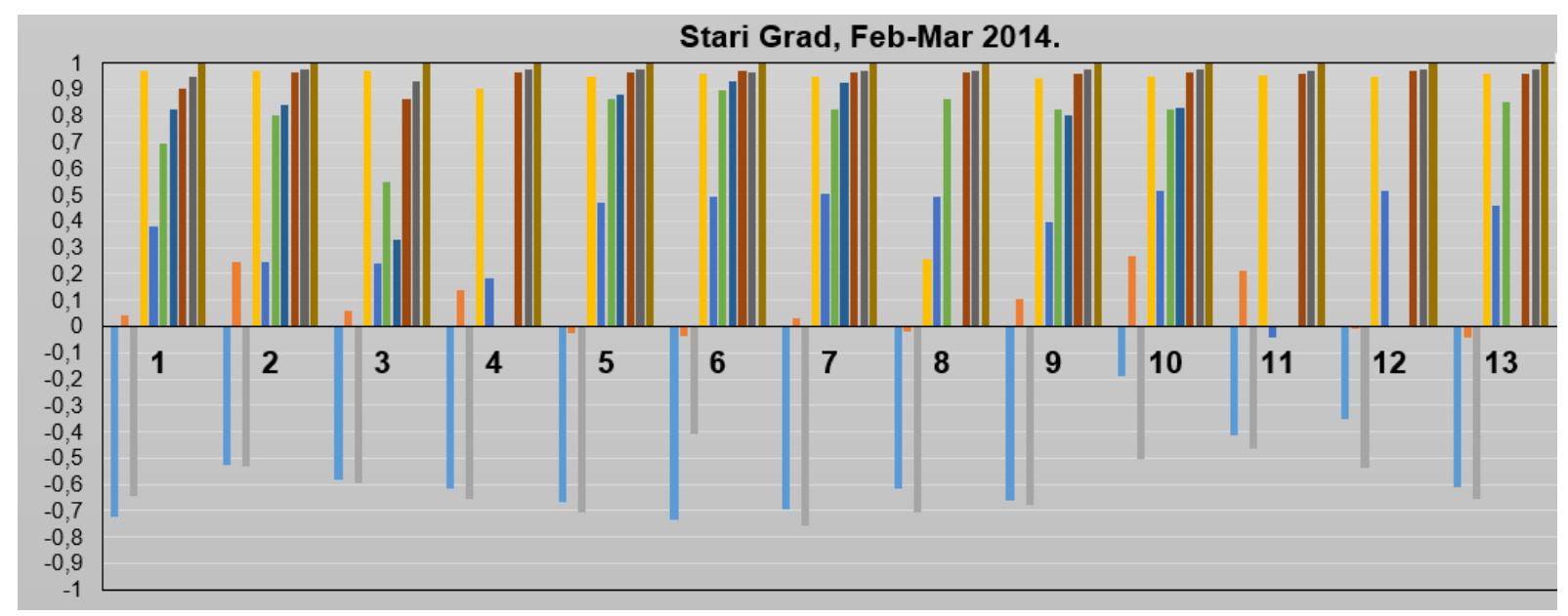

Fig. 2. Pearson coefficient correlation for air pollutants and environmental parameters between lowcost sensors and reference instrument - First campaign: AMS Staii Grad Febraur-March 2014.

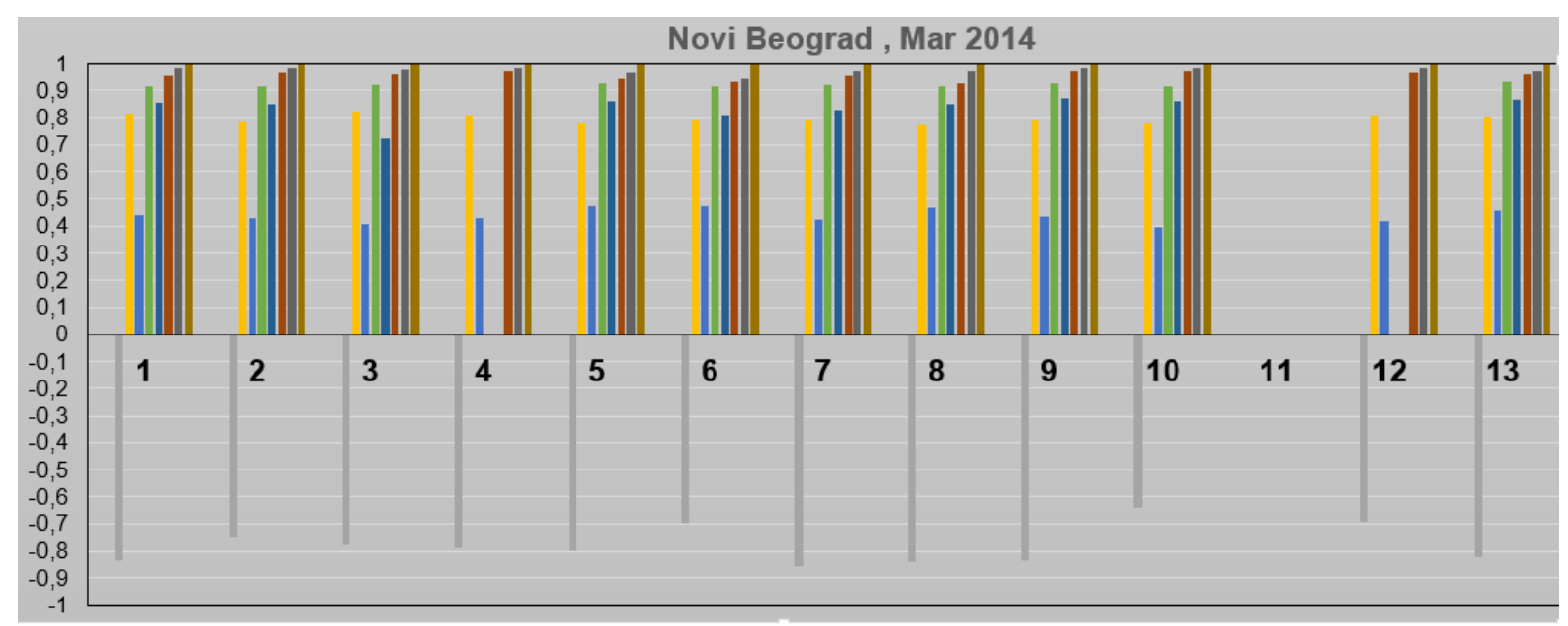

Fig. 3. Pearson coefficient correlation for air pollutants and environmental parameters between lowcost sensors and reference instrument - Second campaign: AMS Novi Beograd March 2014.

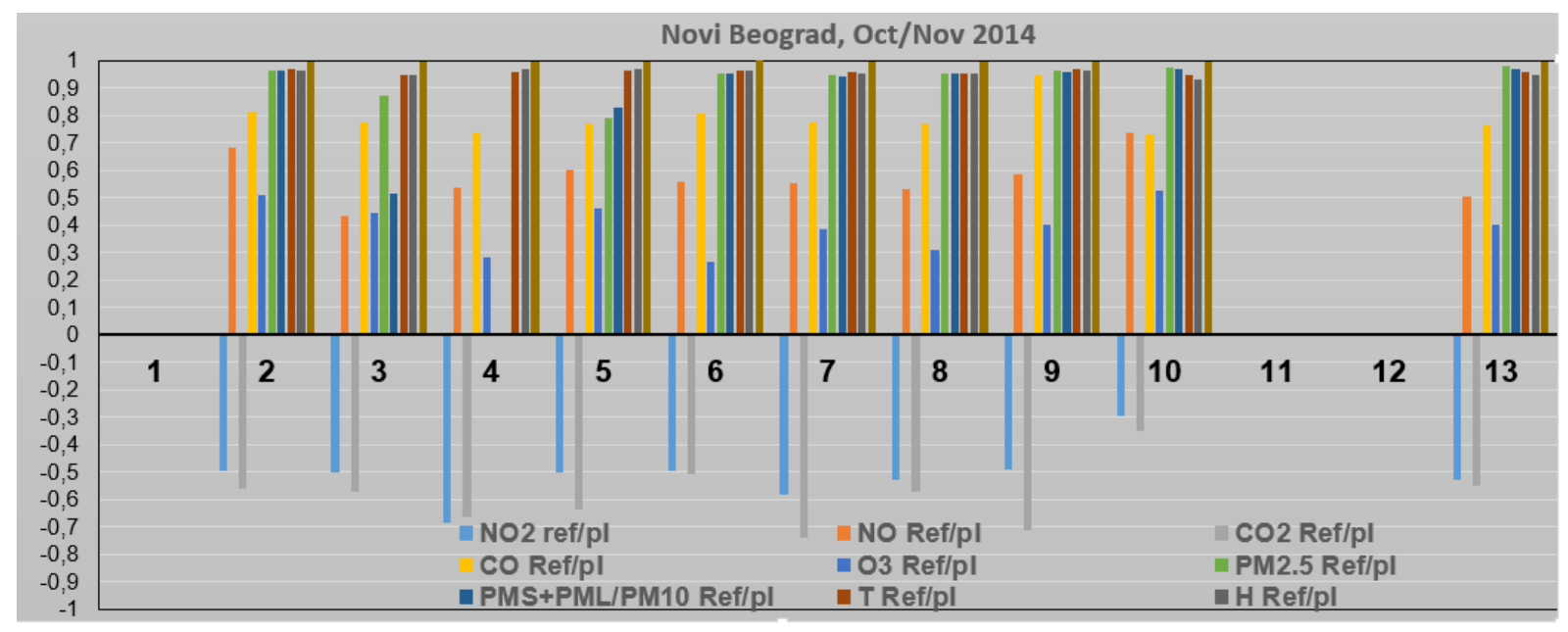

Fig. 4. Pearson coefficient correlation for air pollutants and environmental parameters between lowcost sensors and reference instrument - Last campaign: AMS Novi Beograd October-November 2014. 
Very good agreement is shown between the DYLOS monitors and GRIM devices for particulate matter. With the exception of one DYLOS monitor (no. 3), that had a problem with the ventilator during the first and last campaign, all other low-cost devices correlated very well with the reference instrument with the as $r$ value for both $\mathrm{PM}$ fractions in the range 0.700 0.978 .

CO low-cost sensors show excellent r-values, over 0.900 during the first campaign, but in the range 0.731-0.946 in the later campaigns.

$\mathrm{CO}_{2}$ low cost sensors show the best results during the second campaign, $r$-values were in the range 0.638-0.857. However, during the last campaign 6 of 10 sensors had $r$ below 0.600 .

For ozone Pearson correlation coefficients were generally lower for all sensors and they varied during all campaigns in the range 0.184-0.528.

During the second campaign the reference instrument was not working and results for $\mathrm{NO}_{2}$ and NO sensors were compared in the first and last campaign. There is a big difference in correlation for NO during first and last campaign. Results from the first campaign may be neglected as the supplier of platforms performed some additional improvements between the two campaigns. During the last campaign for NO $r$ was in the range 0.432 0.738 , with an average value of 0.573 . Intra sensors variabilities were highest for $\mathrm{NO}_{2}$, in the range 0.352-0.737, after one sensor that showed much lower correlation with the reference device were excluded. Then the average value of $r$ between low-cost and reference value was 0.600 and 0.534 for the first and last campaign, respectively.

\section{Conclusions and further activities}

In upcoming CITI-SENSE campaigns it is necessary to use the low-cost devices with improved sensors performances that eliminates the influence of $\mathrm{O}_{3}$

Beside that the methodology of collocated calibration and calibration in the field needs to be improved:

a. to apply correction function for air by including environmental parameters data

b. to determine frequency of calibration and life time of sensors

The last, but not the least, we plan to compare results from different available units and focus on intra-sensor performance characteristics and try to determine reliability of each sensor type, with the aim of finding an optimal solution for analyzing and presenting indicative levels of selected pollutants and environmental data in the form that may be usable for citizens.

\section{Acknowledgements}

Presented results have been obtained within the activities that are carried out as part of the ongoing FP7 European project CITI-SENSE (Grant agreement no. 308524).

\section{Reference}

[1] D. Gracia, Robust smoothing of gridded data in one and higher dimensions with missing values, Computational Statistics and Data Analysis 54, 1167-1178 (2010); doi: 10.1016/j.csda.2009.09.020 